\title{
CRECIMIENTO ECONÓMICO Y DEMOGRÁFICO REGIONAL EN COLOMBIA, 1985-2012
}

Luis Armando Galvis*

$\mathrm{E}$ n Colombia, las grandes ciudades priman en el crecimiento económico y demográfico. Bogotá y las demás áreas metropolitanas mantienen un crecimiento relativamente alto. Desde el punto de vista de la economía urbana, ese crecimiento es resultado de la confluencia de varios factores, como la intensidad en el intercambio de ideas, que promueve la innovación y la diversidad urbana y atrae personas (Clark, 2002; Albouy, 2008 y 2010; Carlino y Saiz, 2008). En esta perspectiva, el crecimiento demográfico, en especial la densidad poblacional, es un indicador de la importancia de la intensidad del intercambio o de los contactos cara a cara entre personas de orígenes, culturas y habilidades diferentes (Glaeser, 2011).

Dada la importancia del tema en el país, de mayoría urbana, este artículo estudia la dinámica del crecimiento económico regional y de la densidad poblacional. El objetivo es mostrar cómo ha evolucionado el crecimiento económico y, en especial, la dinámica poblacional desde mediados de los ochenta. En el trabajo se emplean las matrices de transición de Markov tradicionales y su versión espacial, y se muestra que los municipios presentan signos de persistencia en su comportamiento demográfico. Las principales ciudades mantienen su jerarquía

* Economista del Centro de Estudios Económicos Regionales, Banco de la República, sucursal Cartagena, Colombia, [1galviap@ banrep.gov.co]. Una versión anterior aparece en [http://www.banrep.gov.co/publicaciones/pub_ec_reg4.htm]. El autor agradece los comentarios de Adolfo Meisel, Andrés Sánchez, Gerson J. Pérez, Mónica S. Gómez, María Aguilera, Andrea Otero y Karelys Guzmán. Así como la invaluable asistencia de Bladimir Carrillo y Simón Chaves, estudiantes en práctica del CEER, y los comentarios de los evaluadores anónimos de la Revista. Fecha de recepción: 26 de septiembre de 2013, fecha de modificación: 5 de marzo de 2014, fecha de aceptación: 29 de octubre de 2015. Sugerencia de citación: Galvis, L. A. "Crecimiento económico y demográfico regional en Colombia, 1985-2012", Revista de Economía Institucional 17, 33, 2015, pp. 183-201. DOI: http://dx.doi.org/10.18601/01245996.v17n33.08 
y siguen siendo las más densamente pobladas. Los municipios menos prósperos se mantienen relativamente estancados y exhiben bajos niveles de densidad poblacional.

El artículo se divide en cinco secciones. En la primera se hace una revisión teórica de los determinantes del crecimiento urbano. En la segunda se explica la metodología para estudiar la dinámica del crecimiento económico y demográfico. En la tercera se presentan algunas estadísticas del crecimiento económico departamental. La cuarta presenta los resultados de las matrices de transición de Markov tradicionales y las espaciales. La última resume las conclusiones.

\section{CRECIMIENTO URBANO, EXTERNALIDADES Y DENSIDAD DEMOGRÁFICA}

En la literatura sobre economía urbana se plantea que la difusión de conocimientos es una de las principales fuentes de crecimiento económico (Albouy, 2010; Glaeser, 2009): algunos sectores e incluso algunas ciudades crecen porque reciben las externalidades del conocimiento generado en sectores o espacios cercanos. Es decir, crecen algunos sectores por influencia de ideas o conocimientos generados en otros que "se propagan" sobre los primeros ${ }^{1}$. En otras palabras, estos aprovechan esas externalidades, como indicó Marshall (1920), quien analizó por qué las empresas se ubicaban en zonas urbanas y encontró que se debía a las externalidades positivas en forma de difusión de conocimientos provenientes de otras firmas.

Cuando una industria ha escogido una localidad para ubicarse, es probable que permanezca allí largo tiempo, pues son grandes las ventajas que obtienen los que se dedican a la misma industria debido a la proximidad. Los misterios del comercio desaparecen; es como si estuvieran en el aire (ibíd., 225).

Los nuevos conocimientos o ideas que dan origen a otras innovaciones de pensamiento se propagan en forma más expedita en ambientes urbanos que en áreas rurales dispersas (Glaeser, 2011). Este planteamiento no es nuevo y tiene casi un siglo de historia. Por ejemplo, los principios marshallianos resaltan el papel de los flujos de ideas

1 Como ejemplos de este fenómeno cabe citar el de Silicon Valley en San Francisco, donde se consolidó la industria de semiconductores, y el de la Ruta 128 en Boston, que Kenney y von Burg (1999) examinan como clusters donde se refuerza la creación de nuevas firmas que se benefician por la interacción con firmas existentes, y donde después de la Segunda Guerra Mundial se consolidaron empresas como Apple Computer, Cisco Systems, Intel y Sun Microsystems. En esos casos el desarrollo local se basó en el conocimiento y en sus externalidades, que al parecer son el factor clave para su éxito. En vez de un cambio tecnológico exógeno, en estos lugares parece haber un crecimiento endógeno (Romer, 1986) impulsado por los cambios tecnológicos. 
como externalidades que aparecen cuando trabajadores y empresarios comparten pensamientos novedosos. Marshall señaló que "si un hombre inicia una nueva idea, esta es asimilada por otros combinada con sugerencias propias y se convierte en fuente de nuevas ideas" (Marshall 1920, 225).

Por su parte, Jane Jacobs (1969), la autora que revivió esta discusión en el campo de la economía, destacó el contacto persona a persona en las áreas urbanas y señaló que la creencia de que las ciudades surgieron a partir del desarrollo de las zonas rurales es un mito. Jacobs señaló que la producción de algunos bienes comenzó en las ciudades, y que estos luego llegaron a las zonas agrícolas y las ayudaron a crecer y modernizarse. Uno de sus ejemplos es la producción de fertilizantes y maquinaria agrícola que ayudaron a los campesinos a ser más productivos. De ese modo, incluso el "motor del crecimiento" de las zonas rurales se originó en las ciudades, quizá con ayuda de las externalidades del conocimiento que fluyeron entre ciudades y más tarde a las prácticas agrícolas. A veces se encuentra que en las zonas rurales hay fábricas de fertilizantes, plantas de energía y de investigación agrícola, pero según ella esas actividades no se originaron allí sino en las ciudades, donde los nuevos productos, resultado de la innovación, se ponen al servicio de las actividades rurales.

Scitovsky (1954) identificó dos tipos de externalidades: pecuniarias y tecnológicas. Para él, las más importantes para el desarrollo económico y la localización de la actividad económica son las externalidades pecuniarias, que surgen por la interdependencia indirecta entre empresas a causa de las actuaciones de otras empresas, que representan a la creciente demanda de otros productores, o por la reducción de costos y el aumento de los beneficios. Por ejemplo, cuando los beneficios de la empresa A aumentan debido a un incremento de la demanda resultante de la ubicación de la empresa B en la misma ciudad. En el caso de las externalidades tecnológicas, el ejemplo clásico es el efecto directo de un apicultor sobre la producción de frutales.

Autores como Krugman (1991), y luego Fujita, Krugman y Venables (1999), revivieron la discusión iniciada por Marshall sobre las externalidades del conocimiento. Según estos últimos, para Marshall los distritos industriales surgen debido a: (1) las externalidades del conocimiento; (2) las ventajas de la existencia de mercados más amplios para las habilidades especializadas, y (3) los encadenamientos hacia adelante y hacia atrás, que se asocian con mercados locales grandes. Esas tres fuentes de crecimiento están presentes en los sectores económicos y en las ciudades del mundo real, pero los modelos iniciados 
con la nueva geografía económica en general han dejado de lado las dos primeras debido a que son difíciles de modelar, principalmente por falta de datos (Fujita et al., 1999, 5).

Sin embargo, otra línea de investigación se ha enfocado en la localización geográfica de las externalidades, modelando más directamente los flujos de conocimiento: la tradición iniciada por A. Jaffe, M.Trajtenberg y R. Henderson (1993), en adelante JTH. Estos autores dieron inicio a una nueva ola de investigaciones sobre la difusión de conocimientos, usando las citaciones de patentes para medir las externalidades del conocimiento. El argumento es que uno de los factores que explican la localización de las industrias es la posibilidad de aprovechar los flujos de conocimiento. Las citaciones de patentes son una forma de medir esos flujos y saber dónde se originan y a qué sectores benefician.

El trabajo de JTH comenzó como una crítica a Krugman (1991) y revitalizó una discusión que aún prosigue en diversos estudios de economía urbana y regional. Surgió entonces un campo de investigación que sigue la línea de JTH y explora diferentes maneras de vincular la geografía y la actividad económica empleando las patentes y sus citas. Glaeser (2011) retoma algunos de sus planteamientos y señala que la proximidad acelera el flujo de las invenciones más importantes, pues las citas de patentes son más frecuentes en sitios cercanos, es decir, las ideas y el flujo de ideas entre inventores están geográficamente localizados. La mayor densidad de población en las ciudades es un resultado de la búsqueda de fuentes de innovación, porque la creatividad humana es más vigorosa en las zonas urbanas (Glaeser 2011,67).

La innovación y el intercambio de ideas son pues componentes esenciales del crecimiento económico, y son más intensos en las zonas urbanas que en las zonas rurales dispersas. Por ello, en la literatura sobre crecimiento urbano y externalidades del conocimiento la medida de la interacción entre individuos está dada por la densidad demográfica. Esta variable es un indicador de la prosperidad de una ciudad o región que recibe gran atención en el campo de la economía urbana, y es el principal objeto de estudio de este artículo.

\section{MARCO METODOLÓGICO DE LA DINÁMICA DEL CRECIMIENTO}

En las últimas tres décadas, uno de los principales temas de estudio relacionados con el crecimiento económico es el análisis de la convergencia y las disparidades entre regiones, donde sobresalen los trabajos de Abramovitz (1986), Romer (1986), Lucas (1988), Quah (1993, 1996), Magrini (1999) y Barro y Sala-I-Martin (1990), cuyos apor- 
tes han sido muy influyentes. Esos autores estudian la convergencia no condicional, que supone una relación negativa entre el nivel de ingreso per cápita inicial y la tasa de crecimiento de las economías, dados unos rendimientos decrecientes del capital.

Barro (1990), Barro y Sala-I-Martin (1991, 1992) y Mankiw et al. (1992) argumentaron que si los parámetros tecnológicos de un conjunto de economías son diferentes, cada una tendrá un estado estacionario propio en el largo plazo. Es decir, altos niveles de ingreso per cápita implican menores tasas de crecimiento; lo que se llama hipótesis de convergencia condicional y que se ha evaluado estimando regresiones transversales de la tasa de crecimiento sobre el ingreso per cápita inicial, controlando la influencia de variables que determinan el estado estacionario. Quah critica esta forma de estudiar la convergencia, argumentando que las regresiones de corte transversal son poco informativas en el análisis de convergencia, pues nada dicen de la dinámica de la distribución (Quah, 1996, 1369). Además, la relación negativa entre tasa de crecimiento e ingreso per cápita puede ser consistente con la existencia de convergencia (Quah, 1993, 429).

Como método alternativo Quah sugiere emplear cadenas de Markov, que muestran la transición de un estado a otro dentro de un número finito de posibles estados, por ejemplo, en la distribución del ingreso. Un aspecto innovador de este método es que permite identificar la persistencia o reducción de las disparidades, y su probabilidad de ocurrencia, es decir, saber qué tan probable es que un individuo que está en la parte más baja de la distribución en t, siga en ella al cabo de $k$ periodos (también llamados "pasos"). Formalmente, si $P$ es la matriz de transición y $\omega$ el estado de la distribución del ingreso en $t$, la dinámica del sistema en $k$ pasos está dada por:

$\omega^{\mathrm{t}+\mathrm{k}}=P \omega^{\mathrm{t}}$,

Cada elemento de la matriz $P$ muestra la probabilidad de que los individuos ubicados en una parte $i$ de la distribución en $t$ lleguen a una parte $j$ en $t+1$. La inspección de $P$ permite identificar si existe persistencia o convergencia del ingreso. Si $P$ es ergódica, el sistema sigue una distribución estacionaria, y la probabilidad de que una economía ocupe la posición $j$ en la distribución del ingreso en $k$ pasos, dado que se halla en la posición $i$, es igual a la probabilidad de ocupar la posición $j$. En otras palabras, la probabilidad condicional de transición es igual a la probabilidad no condicional (Feller, 1968). En el análisis de convergencia es importante la existencia de estacionariedad en las probabilidades de transición, pues permite saber si la distribución del ingreso converge a un estado estacionario. En su análisis, Quah (1993) 
discretiza la distribución del ingreso en cinco clases mutuamente excluyentes que representan los “estados". Esta elección de cinco clases es arbitraria y se pueden usar otras clasificaciones.

Una alternativa para esa categorización es usar la posición de un área geográfica en un año dado, condicionada a que en el periodo anterior los vecinos de tal unidad estén en una de las posiciones de la distribución (Rey, 2001). Otra opción para incluir los efectos espaciales en las matrices de Markov es categorizar los movimientos de las unidades espaciales de acuerdo con su ubicación en el diagrama de autocorrelación de Moran, el diagrama que relaciona en el eje horizontal la variable estandarizada que se analiza, $Z$, con respecto a su rezago, $W Z$ (ilustración 1 ). $W$ es la matriz de pesos espaciales. En el diagrama de Moran hay cuatro cuadrantes que indican la posición relativa de los valores de $Z$ frente a los de $W Z$, y definen el análisis de los índices locales de análisis espacial (LISA, por sus siglas en inglés). $\mathrm{Si}$ se consideran las parejas de valor propio y valor en el vecindario, en el cuadrante 1 tenemos observaciones de valores altos rodeados de valores altos, (A, A). En el cuadrante 2, valores bajos de la variable $Z$ y altos en su vecindario, (B, A). Los cuadrantes 3 y 4 presentan resultados opuestos. Las observaciones ubicadas en los cuadrantes 1 y 3 están asociadas a la autocorrelación espacial positiva; las que se ubican en los cuadrantes 2 y 4 muestran correlación negativa.

Ilustración 1

Diagrama de dispersión de Moran

$W Z$\begin{tabular}{|c|c|}
\hline $\begin{array}{c}2 \\
(\mathrm{BA})\end{array}$ & $\begin{array}{c}1 \\
(\mathrm{AA})\end{array}$ \\
\cline { 2 - 3 }$\left(\begin{array}{c}3 \\
(\mathrm{BB})\end{array}\right.$ & $\begin{array}{c}4 \\
(\mathrm{AB})\end{array}$ \\
\hline
\end{tabular}

Fuente: elaboración del autor.

En el análisis de la matriz LISA-Markov se tienen en cuenta las transiciones entre los cuadrantes del diagrama de Moran, del periodo t al periodo $\mathrm{t}+1$. Las transiciones no solo dependen de cuál es la dinámica de los movimientos propios de cada municipio sino de qué posición relativa ocupan las observaciones vecinas en el espacio.

El análisis de este tipo de matrices supone que se cumple la propiedad de que las probabilidades de transición son invariantes en el tiempo y, así mismo, que esas probabilidades son independientes de la dimensión espacial. Para este efecto se emplea la prueba chi cuadrado 
que proponen Bickenbach y Bode (2001), que consiste en evaluar si las entradas de dos matrices son iguales entre sí: $\hat{p}_{i j}=\hat{p}_{i j}^{0}$, donde $\hat{p}_{i j}^{0}$ se toma como referencia de comparación:

$Q_{i}^{*}=\sum_{i=1}^{N} \sum_{j \varepsilon F i} \frac{\left(\hat{p}_{i j}=\hat{p}_{i j}^{0}\right)^{2}}{\hat{p}_{i j}^{0}} \sim \chi^{2}\left(f_{i}-1\right)$

donde $F_{i}=\left\{j: \hat{p}_{i j}^{0}>0\right\} ; f_{i}$ es el número entradas en cada una de las filas $i$ que son diferentes de cero en la matriz de referencia.

Esa prueba se adapta para evaluar la independencia espacial y determinar si una o varias submatrices espaciales son diferentes a la matriz general sin condicionar en la dimensión espacial (Rey, 2001). En este caso $\hat{p}_{i j}$ representa las entradas de la matriz condicionada en la dimensión espacial y $\hat{p}_{i j}^{0}$ las de la matriz no condicionada. En ambos casos, si se rechaza la Ho no se cumple la propiedad de Markov, y una matriz general no es suficiente. Por ello se requiere un análisis más detallado, que puede consistir en emplear varios periodos o en condicionar en el espacio.

\section{DINÁMICA DEL INGRESO PER CÁPITA REGIONAL}

La gráfica 1 muestra la relación entre el PIB departamental per cápita y el promedio nacional e incluye los departamentos con cifras superiores al promedio. Los departamentos tradicionalmente importantes se mantienen hasta el final del periodo con cifras mayores que la media: Antioquia, Valle, Cundinamarca y Bogotá. Los demás han surgido en épocas más recientes, en especial debido al auge del sector petrolero; entre ellos se destacan Meta, Santander y los nuevos departamentos.

Bogotá se ha mantenido por encima del promedio, pero su participación se ha reducido debido a que el PIB per cápita de los departamentos donde la actividad petrolera es importante registra una tendencia creciente. Santander, por ejemplo, ha ganado terreno desde comienzos del periodo, y hoy muestra indicadores favorables en varias ramas del sector secundario y de servicios, y no solo en el sector de minería e hidrocarburos (Aguilera, 2013). En este departamento parecen confluir una serie de variables que refuerzan la relación entre prosperidad y densidad demográfica, como las variables institucionales, la capacidad empresarial, la adecuada disposición de mano de obra calificada y con buen nivel de calidad.

Los resultados anteriores muestran una dicotomía entre los departamentos de mayor PIB per cápita y los de la parte baja de la distribución, la cual sugiere una persistencia de la jerarquía con respecto a esta variable. Y esto lo confirman las matrices de transición que se 
muestran en el cuadro 1. Por simplicidad, los grupos de ingreso se dividen por quintiles: pobres, bajos ingresos, ingreso medio, ingreso alto y ricos, P, B, M, A y R. Con esta clasificación Bogotá se mantiene en el grupo de departamentos ricos durante todo el periodo. En el panel A los nuevos departamentos se agregan en una sola unidad y en el panel B aparecen en forma independiente.

Gráfica 1

PIB per cápita departamental/PIB promedio, 1985-2012

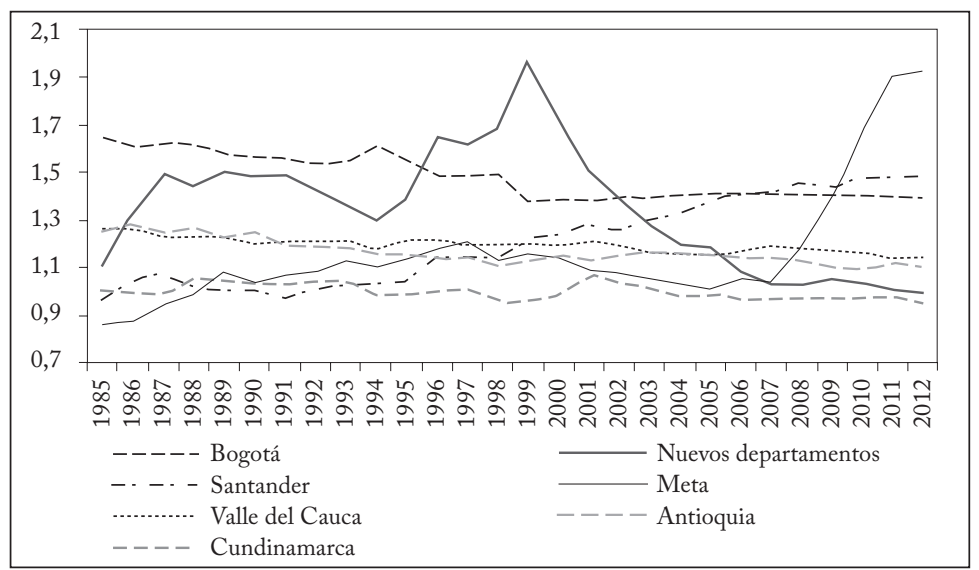

Fuente: Dane, cuentas departamentales, cálculos del autor.

Los resultados del panel A indican que si un departamento estaba en el grupo de pobres al inicio, la probabilidad de que siga en ese grupo al final del periodo es del 92,6\%. Para el grupo de ricos el índice de persistencia es mayor: la probabilidad de seguir en ese grupo en el año $\mathrm{t}+1$ es del 97\%. En el panel B, las probabilidades de permanecer en los extremos son mayores que las de permanecer en los puntos medios de la distribución. Sin embargo, las probabilidades no son estrictamente comparables con las del panel A, porque en el panel B no se agregan los nuevos departamentos en una sola unidad.

En general, los resultados de este ejercicio concuerdan con los de trabajos anteriores, como el de Bonet y Meisel (2007) sobre el ingreso per cápita, y el de González (2011) sobre el PIB per cápita. Los índices de persistencia indican que no hay convergencia del ingreso per cápita, como han documentado otros estudios sobre el país.

Dado que el crecimiento económico está relacionado con la densidad poblacional (Glaeser, 2011), un ejercicio interesante sería analizar la dinámica demográfica durante las últimas décadas, así como se analizó el crecimiento económico. Una ventaja de estudiar 
la dinámica demográfica empleando la densidad poblacional es que se puede hacer un análisis más detallado, a nivel municipal. Pero este ejercicio no se puede realizar porque no hay datos disponibles de la actividad económica a nivel municipal.

Cuadro 1

Matrices de transición de Markov con base en el PIB per cápita, 1985-2012 Panel A. Periodo 1985-2012

\begin{tabular}{|c|c|c|c|c|c|c|}
\hline & \multicolumn{5}{|c|}{ Grupo de ingreso en el año $t+1$} \\
\hline & & $\mathrm{P}$ & B & M & $\mathrm{A}$ & $\mathrm{R}$ \\
\hline \multirow{5}{*}{$\begin{array}{l}\text { Grupo de } \\
\text { ingreso en el } \\
\text { año } t\end{array}$} & $\mathrm{P}$ & 92,6 & 7,4 & 0,0 & 0,0 & 0,0 \\
\hline & $\mathrm{B}$ & 7,4 & 81,5 & 11,1 & 0,0 & 0,0 \\
\hline & $\mathrm{M}$ & 0,0 & 10,4 & 81,5 & 8,1 & 0,0 \\
\hline & A & 0,0 & 0,7 & 7,4 & 88,9 & 3,0 \\
\hline & $\mathrm{R}$ & 0,0 & 0,0 & 0,0 & 3,0 & 97,0 \\
\hline
\end{tabular}

Panel B. Periodo 1990-2012

\begin{tabular}{|c|c|c|c|c|c|c|}
\hline & \multicolumn{5}{|c|}{ Grupo de ingreso en el año $t+1$} \\
\hline & & $\mathrm{P}$ & B & M & A & $\mathrm{R}$ \\
\hline & $\mathrm{P}$ & 91,6 & 7,8 & 0,6 & 0,0 & 0,0 \\
\hline Grupo de & B & 9,1 & 82,6 & 8,3 & 0,0 & 0,0 \\
\hline ingreso en el & M & 0,6 & 5,8 & 83,1 & 10,4 & 0,0 \\
\hline año $t$ & A & 0,0 & 0,8 & 10,6 & 85,6 & 3,0 \\
\hline & $\mathrm{R}$ & 0,0 & 0,6 & 0,0 & 1,9 & 97,4 \\
\hline
\end{tabular}

Nota: en el panel A los nuevos departamentos se agregan en una sola unidad. En el panel B, las cifras disponibles permiten desagregar cada departamento. Las pruebas de homogeneidad, empleando el estadístico Q*, sugieren que no existe cambio estructural, y muestran un quiebre en el año 2000. De todas formas, Bickenbach y Bode (2002) recomiendan el análisis agrupado cuando hay pocas observaciones.

Fuente: Dane, cálculos del autor.

\section{DINÁMICA DE LA DENSIDAD POBLACIONAL MUNICIPAL}

En Bogotá, la ciudad de mayor aglomeración poblacional, residían 7.571.345 personas en 2012, el 16,3\% del total. La consolidación de la capital como urbe principal en términos económicos y demográficos es resultado de varios factores que se estudian en detalle en Galvis (2014). Entre 1985 y 1993 su crecimiento demográfico promedio anual fue de un 3,1\%. En el siguiente periodo censal, 1993-2005, su población creció a una tasa anual cercana al $2 \%$. Las principales ciudades muestran notables crecimientos demográficos, especialmente si se comparan con las áreas rurales que han expulsado población. Para ver cómo ha evolucionado la densidad poblacional de los municipios, a continuación se analiza la dinámica del crecimiento de la densidad poblacional usando las matrices de transición de Markov.

\section{MATRICES DE MARKOV TRADICIONALES}

De acuerdo con los resultados anteriores, se esperaría encontrar una polarización del país que concentra la población en las ciudades 
capitales y, en especial, en Bogotá. Para comprobarlo se replica el análisis de la dinámica del crecimiento económico, pero aplicado a la densidad poblacional. En un análisis similar del tamaño poblacional de China e India, Schaffar y Dimou (2012) y, de países de los Balcanes, Dimou y Schaffar (2009) encuentran que las ciudades de estos países no siguen el patrón que predice la Ley de Zipf. En India la dinámica demográfica tiene un carácter dual: un gran número de ciudades pequeñas y un bajo número de áreas metropolitanas enormes. En Colombia, los cálculos de la densidad poblacional municipal arrojan los resultados que se presentan en el cuadro 2: una alta persistencia de la jerarquía de tamaños poblacionales, sobre todo en los quintiles más bajos y altos de la distribución. Bogotá, como era de esperar, se mantiene en el quintil de mayor densidad poblacional. En el tercer quintil se presenta la mayor movilidad. E1 equilibrio que se alcanza en el largo plazo, asociado a los valores del estado estacionario, muestra que las densidades se distribuirían homogéneamente entre quintiles.

Los resultados de las dos matrices inferiores indican que en el segundo periodo hubo mayor movilidad que en el primero, en especial en el grupo de municipios de los quintiles segundo y tercero. Examinando dónde ha habido mejoras en la posición que los municipios ocupaban antes, se encuentra que han ocurrido en municipios cercanos entre sí, como muestra el mapa 1. Igual ocurre con las pérdidas en la jerarquía de densidad, que suelen ocurrir en municipios alejados de la capital departamental. Esta última normalmente tiene mejores perspectivas en cuanto se refiere a densidad poblacional, por las ventajas que obtienen las personas que allí se localizan. En otras palabras, los municipios ganadores son casi siempre las capitales departamentales, o sus vecinos. Este patrón también se observa en los índices de pobreza, medidos por el índice de NBI (Galvis, 2009). En este caso, en el Caribe Continental, las zonas menos prósperas corresponden a municipios alejados de la capital, en su mayoría rurales, con mala dotación de servicios básicos. Cuanto más lejanos de las capitales departamentales, mayor es su grado de pobreza. Por ejemplo, en 1993 la correlación entre distancia de la cabecera municipal a la capital y carencia de servicios básicos, medida por el NBI, era del 52\%, y en 2005 del 51\%.

Los resultados anteriores dan argumentos para estudiar las relaciones de persistencia o movilidad de la dinámica poblacional en los municipios desde una perspectiva espacial, es decir, teniendo en cuenta su ubicación relativa. Ello se logra involucrando las matrices 
espaciales en el análisis de las matrices de transición tradicionales, como propone Rey (2001).

Cuadro 2

Matrices de transición de Markov de la densidad poblacional, 1985-2005

\begin{tabular}{|c|c|c|c|c|c|c|}
\hline \multicolumn{2}{|l|}{$1985-2005$} & \multicolumn{5}{|c|}{ Quintil en el año $t+1$} \\
\hline & & 1 & 2 & 3 & 4 & 5 \\
\hline \multirow{5}{*}{ Quintil en el año $t$} & 1 & 91,8 & 7,7 & 0,5 & 0,0 & 0,0 \\
\hline & 2 & 8,0 & 78,2 & 13,8 & 0,0 & 0,0 \\
\hline & 3 & 0,2 & 13,8 & 70,1 & 15,8 & 0,0 \\
\hline & 4 & 0,0 & 0,2 & 14,8 & 76,0 & 9,0 \\
\hline & 5 & 0,0 & 0,0 & 0,7 & 8,3 & 91,0 \\
\hline \multicolumn{2}{|l|}{ Estado estacionario } & 20 & 20 & 20 & 20 & 20 \\
\hline \multicolumn{2}{|l|}{$1985-1993$} & 1 & 2 & 3 & 4 & 5 \\
\hline \multirow{5}{*}{ Quintil en el año t } & 1 & 92,8 & 6,8 & 0,5 & 0,0 & 0,0 \\
\hline & 2 & 7,3 & 83,5 & 9,2 & 0,0 & 0,0 \\
\hline & 3 & 0,0 & 9,2 & 77,2 & 13,6 & 0,0 \\
\hline & 4 & 0,0 & 0,5 & 13,1 & 79,1 & 7,3 \\
\hline & 5 & 0,0 & 0,0 & 0,0 & 7,3 & 92,7 \\
\hline \multicolumn{2}{|l|}{ Estado estacionario } & 20 & 20 & 20 & 20 & 20 \\
\hline \multicolumn{2}{|l|}{ 1993-2005 } & 1 & 2 & 3 & 4 & 5 \\
\hline \multirow{5}{*}{ Quintil en el año $t$} & 1 & 90,8 & 8,7 & 0,5 & 0,0 & 0,0 \\
\hline & 2 & 8,7 & 72,8 & 18,4 & 0,0 & 0,0 \\
\hline & 3 & 0,5 & 18,4 & 63,1 & 18,0 & 0,0 \\
\hline & 4 & 0,0 & 0,0 & 16,5 & 72,8 & 10,7 \\
\hline & 5 & 0,0 & 0,0 & 1,5 & 9,2 & 89,3 \\
\hline \multicolumn{2}{|l|}{ Estado estacionario } & 20 & 20 & 20 & 20 & 20 \\
\hline
\end{tabular}

Pruebas de homogeneidad, $Q^{*}$

\begin{tabular}{lccc}
\hline Ho: & Grados de libertad & $\mathrm{Q}^{*}$ & Valor $\mathrm{p}$ \\
\hline Ho: $\hat{p}_{i(1993-2005)}=\hat{p}_{i j(1985-1993)} \mid \mathrm{i}=1$ & 2 & 0,97 & 0,61 \\
Ho: $\hat{p}_{i j(1993-2005)}=\hat{p}_{i j(1985-1993)} \mid \mathrm{i}=2$ & 2 & 13,23 & 0,00 \\
Ho: $\hat{p}_{i j(1993-2005)}=\hat{p}_{i j(1985-1993)} \mid \mathrm{i}=3$ & 3 & 19,16 & 0,00 \\
Ho: $\hat{p}_{i j(1993-2005)}=\hat{p}_{i j(1985-1993)} \mid \mathrm{i}=4$ & 2 & 4,80 & 0,09 \\
Ho: $\hat{p}_{i j(1993-2005)}=\hat{p}_{i j(1985-1993)} \mid \mathrm{i}=5$ & 2 & 4,11 & 0,13 \\
Ho: $\hat{p}_{i j(1993-2005)}=\hat{p}_{i j(1985-1993)}$ & 11 & 42,26 & 0,00 \\
\hline
\end{tabular}

Nota: en los cálculos se tomaron los años censales 1985, 1993 y 2005.

Fuente: Dane, cálculos del autor.

\section{MATRICES ESPACIALES DE MARKOV}

Siguiendo a Schaffar y Dimou (2012), y adoptando la propuesta de Rey (2001) para incorporar la dimensión espacial en el análisis de la dinámica poblacional, se obtienen algunas ventajas relativas con respecto al análisis tradicional de las matrices de Markov. Por ejemplo, se puede saber si la probabilidad de permanecer en una categoría alta, a condición de que los vecinos también estén en una categoría alta, es diferente si los vecinos están en puntos medios o bajos de la distribución. El cuadro 3 muestra los resultados de este ejercicio. 


\section{Mapa 1}

Cambios en el ranking de la densidad de población municipal

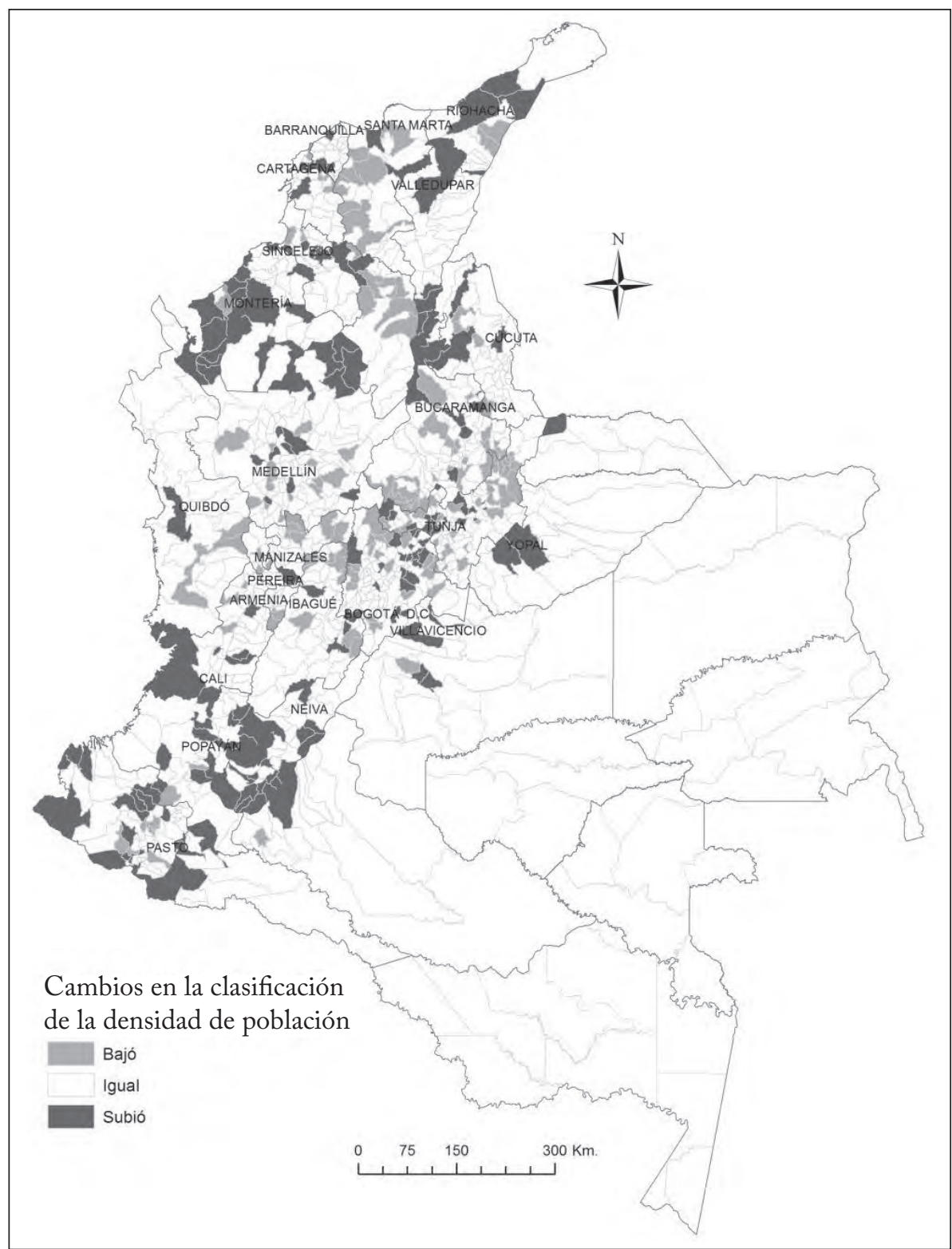

Fuente: Dane, elaboración del autor.

Entre los resultados del análisis espacial de Markov cabe destacar que la probabilidad de que un municipio poco denso se mantenga en esa situación, dado que sus vecinos comparten esa característica, es 
del 98\%. Si los vecinos están en el segundo quintil de la distribución de densidad poblacional, esa probabilidad es del 91,4\%, pero baja al $87 \%$ si están en el tercer quintil. En el otro extremo de la distribución se encuentran los municipios de mayor densidad, cuya probabilidad de seguir siendo altamente densos es del $88,2 \%$ si los vecinos también están en el último quintil de densidad. Si estos se ubican en los quintiles tercero o cuarto, esas probabilidades se reducen al 76,3\% y al 77,5\%, respectivamente. La comparación con las probabilidades de permanecer sobre la diagonal de persistencia lleva a concluir que, una vez se condicionan por la localización de los municipios en el espacio, las probabilidades de transición cambian y muestran un panorama diferente al del análisis tradicional (cuadro 2).

Cuadro 3

Matrices de transición de Markov espaciales para la densidad poblacional, 1985-2005

\begin{tabular}{|c|c|c|c|c|c|c|c|}
\hline & & & \multicolumn{5}{|c|}{ Quintil en el año $t+1$} \\
\hline $\begin{array}{l}\text { til del } \\
\text { rezago } \\
\text { espacial }\end{array}$ & & & 1 & 2 & 3 & 4 & 5 \\
\hline \multirow[t]{5}{*}{ p } & \multirow{5}{*}{$\begin{array}{l}\text { Quintil en } \\
\text { el año } t\end{array}$} & 1 & 98,0 & 2,0 & 0,0 & 0,0 & 0,0 \\
\hline & & 2 & 17,5 & 75,0 & 7,5 & 0,0 & 0,0 \\
\hline & & 3 & 0,0 & 51,7 & 34,5 & 13,8 & 0,0 \\
\hline & & 4 & 0,0 & 0,0 & 0,0 & 83,3 & 16,7 \\
\hline & & 5 & 0,0 & 0,0 & 0,0 & 0,0 & 100,0 \\
\hline \multirow{5}{*}{2} & \multirow{5}{*}{$\begin{array}{l}\text { Quintil en } \\
\text { el año } t\end{array}$} & 1 & 91,4 & 7,1 & 1,4 & 0,0 & 0,0 \\
\hline & & 2 & 20,5 & 75,5 & 4,0 & 0,0 & 0,0 \\
\hline & & 3 & 0,0 & 32,7 & 63,7 & 3,5 & 0,0 \\
\hline & & 4 & 0,0 & 2,7 & 43,2 & 54,1 & 0,0 \\
\hline & & 5 & 0,0 & 0,0 & 0,0 & 4,6 & 95,5 \\
\hline \multirow{5}{*}{3} & \multirow{5}{*}{$\begin{array}{l}\text { Quintil en } \\
\text { el año t }\end{array}$} & 1 & 87,1 & 12,9 & 0,0 & 0,0 & 0,0 \\
\hline & & 2 & 12,9 & 82,8 & 4,3 & 0,0 & 0,0 \\
\hline & & 3 & 0,6 & 31,7 & 63,3 & 4,4 & 0,0 \\
\hline & & 4 & 0,0 & 2,9 & 39,1 & 54,3 & 3,8 \\
\hline & & 5 & 0,0 & 0,0 & 2,5 & 20 & 77,5 \\
\hline \multirow{5}{*}{4} & \multirow{5}{*}{$\begin{array}{l}\text { Quintil en } \\
\text { el año } t\end{array}$} & 1 & 100 & 0,0 & 0,0 & 0,0 & 0,0 \\
\hline & & 2 & 23,1 & 71,2 & 5,8 & 0,0 & 0,0 \\
\hline & & 3 & 1,7 & 22,0 & 72,9 & 3,4 & 0,0 \\
\hline & & 4 & 0,0 & 0,0 & 31,1 & 66,7 & 2,3 \\
\hline & & 5 & 0,0 & 0,0 & 2,2 & 21,6 & 76,3 \\
\hline \multirow{5}{*}{5} & \multirow{5}{*}{$\begin{array}{l}\text { Quintil en } \\
\text { el año } t\end{array}$} & 1 & 100 & 0,0 & 0,0 & 0,0 & 0,0 \\
\hline & & 2 & 6,7 & 93,3 & 0,0 & 0,0 & 0,0 \\
\hline & & 3 & 0,0 & 13,2 & 79,0 & 7,9 & 0,0 \\
\hline & & 4 & 0,0 & 0,0 & 17,6 & 76,8 & 5,6 \\
\hline & & 5 & 0,0 & 0,0 & 1,3 & 10,6 & 88,2 \\
\hline
\end{tabular}

Nota: en los cálculos se tomaron los años censales 1985, 1993 y 2005.

Fuente: cálculos del autor.

Las pruebas de independencia de los movimientos de la densidad poblacional con respecto a los movimientos de su rezago espacial arrojan un estadístico chi cuadrado de 97,08 con un valor p de 0,093. Esto 
indica que, con los niveles de confianza usuales del 5\%, la matriz global de transiciones, sin considerar la dependencia espacial, no muestra resultados diferentes a los que condiciona la ubicación espacial de las unidades estudiadas. Con niveles de confianza menores, del 10\%, estos resultados se pueden complementar con el análisis espacial de la matriz LISA-Markov, como se verá más adelante.

\section{MATRICES DE TRANSICIÓN LISA-MARKOV}

Otra manera de hacer el análisis espacial con las herramientas de las matrices de transición de Markov es utilizar la definición de clusters locales del análisis LISA. En este caso, la densidad poblacional no se categoriza por quintiles, sino que los clusters locales se clasifican bien sea en zonas de alta densidad rodeadas por vecinos de alta densidad poblacional, (Alto-Alto), o áreas de gran atracción con potencial de demanda de ciertos bienes y servicios; o zonas deprimidas, con baja densidad de población, rodeadas de municipios de baja densidad poblacional, (Bajo-Bajo). Por último se establecen también los cruces de sitios de baja densidad rodeados de alta, y viceversa.

Esta categorización es útil para analizar las diferencias de comportamiento demográfico entre una perspectiva espacial y una donde el espacio no importa. Este tipo de análisis aporta evidencia acerca de la idea de que para efectos de planeación se necesitan políticas "ciegas", como propone uno de los últimos informes de desarrollo mundial (Banco Mundial, 2009). Este informe plantea que como la población mundial tiende a ubicarse en las ciudades, en zonas densas, no se necesitan políticas regionales.

Los resultados del análisis LISA-Markov, que se presentan en el cuadro 4, muestran que los municipios tienden a permanecer en sus posiciones de densidad poblacional, condicionadas a la posición de sus vecinos ${ }^{2}$. Así, la probabilidad de que un municipio permanezca en el cuadrante 1 (Alto-Alto), dado que sus vecinos están en la misma posición, es del 83,2\%. En el cuadrante 3 del diagrama de Moran estarían los municipios que tenían baja densidad poblacional en el periodo t, y que estaban rodeados de vecinos en situación similar. La probabilidad de permanecer en esa posición en $t+1$ es del 99\%. Este cuadrante es el de mayor persistencia en la distribución de densidad poblacional. La diferencia en los índices de persistencia sugiere que es más fácil que un municipio próspero, por su densidad, caiga de jerarquía, a que uno relativamente deprimido ascienda de posición.

${ }^{2}$ Como la prueba chi cuadrado da un valor $\mathrm{p}$ de 0,00 se puede concluir que la dinámica de la densidad no es independiente de la de sus rezagos a nivel local. 
Cuadro 1

Matrices de transición LISA-Markov de densidad poblacional, 1985-2005

\begin{tabular}{|c|c|c|c|c|c|}
\hline & \multicolumn{4}{|c|}{ Quintil en el año $t+1$} \\
\hline & & $(\mathrm{A}, \mathrm{A})$ & $(\mathrm{B}, \mathrm{A})$ & $(\mathrm{B}, \mathrm{B})$ & $(\mathrm{A}, \mathrm{B})$ \\
\hline \multirow{4}{*}{ Quintil en el año $t$} & $(\mathrm{~A}, \mathrm{~A})$ & 83,2 & 8,4 & 3,7 & 4,7 \\
\hline & $(\mathrm{B}, \mathrm{A})$ & 2,5 & 86,4 & 11,1 & 0,0 \\
\hline & $(\mathrm{B}, \mathrm{B})$ & 0,1 & 0,5 & 99,0 & 0,5 \\
\hline & $(\mathrm{A}, \mathrm{B})$ & 1,4 & 0,0 & 15,5 & 83,1 \\
\hline \multicolumn{2}{|l|}{ Estado estacionario } & 1,2 & 3,9 & 92,0 & 2,9 \\
\hline \multicolumn{6}{|l|}{ 1985-1993 } \\
\hline \multirow{5}{*}{ Quintil en el año t } & & $(\mathrm{A}, \mathrm{A})$ & $(\mathrm{B}, \mathrm{A})$ & $(\mathrm{B}, \mathrm{B})$ & $(\mathrm{A}, \mathrm{B})$ \\
\hline & $(\mathrm{A}, \mathrm{A})$ & 92,6 & 4,2 & 2,1 & 1,1 \\
\hline & $(\mathrm{B}, \mathrm{A})$ & 1,6 & 95,1 & 3,3 & 0,0 \\
\hline & $(\mathrm{B}, \mathrm{B})$ & 0,0 & 0,4 & 99,3 & 0,3 \\
\hline & $(\mathrm{A}, \mathrm{B})$ & 0,0 & 0,0 & 4,3 & 95,7 \\
\hline \multicolumn{2}{|l|}{ Estado estacionario } & 1,9 & 8,5 & 83,9 & 5,7 \\
\hline \multicolumn{6}{|l|}{ 1993-2005 } \\
\hline & $(\mathrm{A}, \mathrm{A})$ & $(\mathrm{B}, \mathrm{A})$ & $(\mathrm{B}, \mathrm{B})$ & $(\mathrm{A}, \mathrm{B})$ \\
\hline \multirow{4}{*}{ Quintil en el año $t$} & $(\mathrm{~A}, \mathrm{~A})$ & 81,1 & 7,8 & 4,4 & 6,7 \\
\hline & $(\mathrm{B}, \mathrm{A})$ & 2,4 & 82,9 & 14,6 & 0,0 \\
\hline & $(\mathrm{B}, \mathrm{B})$ & 0,0 & 0,1 & 99,5 & 0,4 \\
\hline & $(\mathrm{A}, \mathrm{B})$ & 1,4 & 0,0 & 20,0 & 78,6 \\
\hline \multicolumn{2}{|l|}{ Estado estacionario } & 0,3 & 0,9 & 97,0 & 1,9 \\
\hline
\end{tabular}

Nota: en los cálculos se tomaron los años censales 1985, 1993 y 2005.

Fuente: Dane, cálculos del autor.

Cuando se examina la distribución en el estado estacionario se encuentra que en el largo plazo los municipios en su mayoría tienden a localizarse en el cuadrante 3 (Bajo-Bajo), y unos pocos en el cuadrante 1 (Alto-Alto). En este último cuadrante se ubican Bogotá y las principales áreas metropolitanas. Este resultado es similar al que se mencionó respecto de India: una estructura dual con un gran número de municipios pequeños y un bajo número de áreas metropolitanas grandes que concentran la gran mayoría de la población. Este patrón dual y la distribución espacial de sus componentes se representan en el mapa 2.

En este mapa se clasifica la densidad de población según las desviaciones estándar respecto de la media municipal. En el mapa se resaltan las capitales más densamente pobladas y se aprecia la estructura dual o polarizada de la distribución de la población del país. Tan solo en las 23 capitales de los antiguos departamentos se concentra más del $57 \%$ de la población urbana, en un área que solo cubre el 2,7\% del territorio nacional.

\section{CONCLUSIONES}

E1 desarrollo económico del país en las últimas décadas se caracteriza por una polarización regional. Las ciudades principales han ganado 


\section{Mapa 2}

Distribución de la densidad de población, 2012

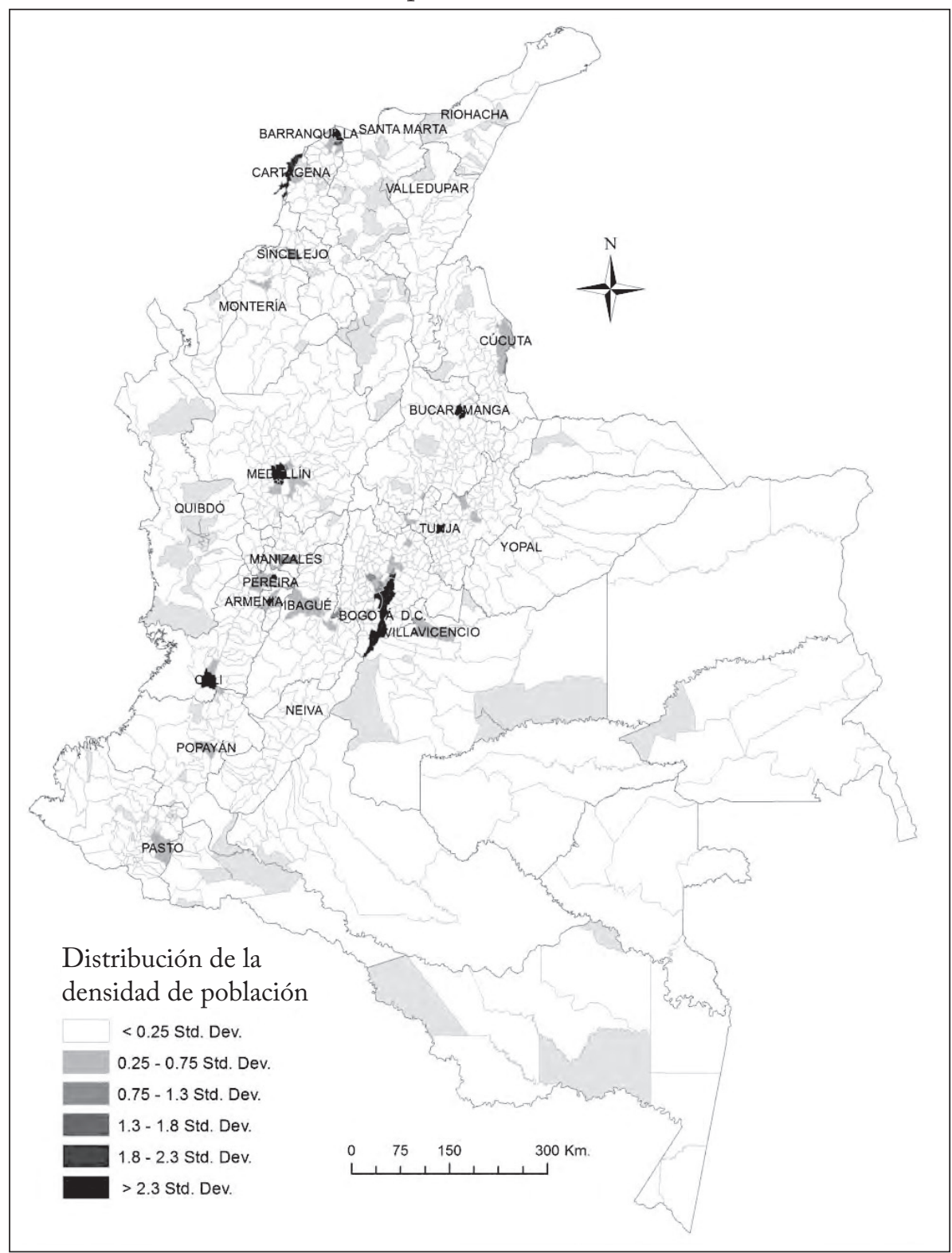

Fuente: Dane, elaboración del autor.

terreno en los aspectos económicos y demográficos. Este fenómeno puede ser favorable porque puede generar ahorros, por ejemplo, en la provisión de servicios a la población, como educación, salud y sanea- 
miento. No obstante, esa polarización trae consigo algunos problemas sobre los cuales hay que reflexionar. Así como la mayor densidad de población en las ciudades favorece el intercambio de ideas, también crea condiciones para que se deteriore la calidad de vida a causa de la congestión vehicular y los mayores índices de delincuencia. Por ello, pese a que la localización y la concentración de la población en los centros urbanos favorecen la planeación regional y urbana en algunos aspectos, también exigen prestar atención a los efectos indeseables de la concentración de la población en las urbes, especialmente en la capital, la cual alberga el 16\% del total.

E1 análisis del crecimiento demográfico lleva a concluir que la dimensión espacial debe estar presente en todo estudio socioeconómico, porque lo que ocurre en un municipio o ciudad no es ajeno a lo que pasa en su vecindario. En ese sentido, es claro que las políticas deben tener un componente regional, más aún en un país donde la abrupta geografía obstaculiza la conexión espacial entre ciudades y regiones. Por último, se debe destacar que el crecimiento demográfico y la densidad poblacional son importantes para el crecimiento económico, pero no son suficientes. Las grandes ciudades también atraen pobreza y para superarla es necesario brindar oportunidades para la movilidad social.

\section{REFERENCIAS BIBLIOGRÁFICAS}

1. Abramovitz, M. "Catching up, forging ahead and falling behind", Journal of Economic History 46, 2, 1986, pp. 385-406.

2. Albouy, D. "Are big cities bad places to live? Estimating quality of life across metropolitan areas”, NBER working paper 14472, 2008.

3. Albouy, D. "What are cities worth? Land rents, local productivity, and the capitalization of amenity values", NBER working paper 14981, 2010.

4. Aguilera, M. "Bucaramanga: capital humano y crecimiento económico", Documentos de trabajo sobre economía regional 180, Banco de la República, 2013.

5. Barro, R. J., y X. Sala-i-Martin. "Convergence", Journal of Political Economy 100, 1992, pp. 223-251.

6. Bickenbach, F. y E. Bode. "Markov or not Markov - This should be a question", Kiel Institute of World Economics, working paper 1086, 2001.

7. Bonet, J. y A. Meisel. "Polarización del ingreso per cápita departamental en Colombia, 1975-2000”, Ensayos sobre Politica Económica 25, 54, 2007, pp. 12-43.

8. Dimou M. y A. Schaffar. "Urban hierarchies and city-growth in the Balkans”, Urban Studies 46, 13, 2009, pp. 2891-2906.

9. Carlino, G. y A. Saiz. "City beautiful", Federal Reserve Bank of Philadelphia, Research Department, working paper 0822, 2008. 
10. Clark, T., R. Lloyd et al. "Amenities drive urban growth", Journal of Urban Affairs 24, 5, 2002, pp. 493-515.

11. Feller, W. An introduction to probability theory and its applications, Nueva York, Wiley and Sons, 1968.

12. Fujita, M.; P. Krugman y A. J. Venables. The spatial economy: Cities, regions, and international trade, Cambridge, Mass., MIT Press, 1999.

13. Galvis, L. "Geografía económica del Caribe Continental”, Documentos de trabajo sobre economía regional 119, Banco de la República, 2009.

14. Galvis, L. “¿E1 triunfo de Bogotá?: desempeño reciente de la ciudad capital”, L. Galvis, ed., Economía de las grandes ciudades en Colombia: seis estudios de caso, Bogotá, Banco de la República, 2014.

15. Gilbert, A. "Land, housing, and infrastructure in Latin America's major cities", A. Gilbert, ed., The mega-city in Latin America, Nueva York, United Nations University Press, 1996.

16. Glaeser, E. y J. Gottlieb. "The wealth of cities: Agglomeration economies and spatial equilibrium in the United States", Journal of Economic Literature 47, 4, 2009, pp. 983-1028.

17. Glaeser, E. Triumph of the city: How our greatest invention makes us richer, smarter, greener, healthier, and happier, Nueva York, Penguin Press, 2011.

18. González, N. “¿Otra vez? Una sencilla visión de la convergencia económica en los departamentos de Colombia: 1975-2005”, Archivos de Economía 384, 2011.

19. Jacobs, J. The economy of cities, Nueva York, Vintage, 1969.

20. Jaffe, A. B.; M. Trajtenberg y R. Henderson. “Geographic localization of knowledge spillovers as evidenced by patent citations", Quarterly Journal of Economics 108, 3, 1993, pp. 577-598.

21. Kenney, M. y U. von Burg. “Technology, entrepreneurship and path dependence: industrial clustering in Silicon Valley and Route 128", Industrial and corporate change 8, 1, 1999, pp. 67-103.

22. Krugman, P. "Increasing returns and economic geography", Journal of Political Economy 99, 3, 1991, pp. 483-499.

23. Lucas Jr., R. "On the mechanics of economic development", Journal of Monetary Economics 22, 1, 1988, pp. 3-42.

24. Magrini, S. "The evolution of income disparities among the regions of the European Union", Regional Science and Urban Economics 29, 2, 1999, pp. 257-281.

25. Mankiw, G.; D. Romer y R. Weil. "A contribution to the empirics of economic growth”, Quarterly Journal of Economics 108, 1992, pp. 503-530.

26. Marshall, A. Principles of economics, Londres, Macmillan, 1920.

27. Quah, D. "Galton's Fallacy and the convergence hypothesis", Scandinavian Journal of Economics 95, 1993, pp. 427-443.

28. Quah, D. "Empirics for economic growth and convergence", European Economic Review 40, 6, 1996, pp. 1353-1375.

29. Rey, S. "Spatial empirics for economic growth and convergence", Geographical Analysis 33, 3, 2001, pp. 195-214.

30. Romer, P. M. "Endogenous technological change", Journal of Political Economy 98, 5, 1990, pp. 71-102. 
31. Romer, P. M. “Increasing returns and long-run growth", Journal of Political Economy, 94, 5, 1986, pp. 1002-1037.

32. Schaffar, A. y M. Dimou. "Rank-size city dynamics in China and India, 1981-2004”, Regional Studies 46, 6, 2012, pp. 707-721.

33. Scitovsky, T. "Two concepts of external economies", Journal of Political Economy 62, 2, 1954, pp. 143-151. 